\title{
Experience of using antiepileptic drugs in children with Autism spectrum disorder.
}

Zaytsev's Psychoneurological Center Itd. Ligivsky prospect, 50 liter F Russian Federation, Saint-Petersburg Dmitry Zaytsev, Ilya Zaytsev - psychiatry Nikolay Titov - neurology

Objectives. Autism spectrum disorder (ASD) is one of the most frequent neurodevelopmental disorder in pediatric psychiatry with prevalence of about 1 in 68 children. It is characterized by persistent deficits in social, verbal and nonverbal communication, social interaction and restricted, repetitive patterns of behavior, interests or activities. Treatment resistance induce psychiatrists to find new methods of diagnostic. Aim to study bioelectric activity of the brain for presence of epileptiform discharges and its influence on management tactics in children with ASD.

Material and methods. We analyzed 50 children (212 years old) with ASD (DSM-V classification) without epileptic seizures in history. All children were examined by a psychiatrist, a neurologist and a speech therapist at least twice. All children were diagnosed with long-term EEG monitoring twice. Group I: 24 children aged 2-12 years with period of normal development and subsequent development regress. Group II: 26 children aged 2-8 years without period of normal development.

Results. 16 of $24(66,7 \%)$ patients in group I and 17 of $26(62.5 \%)$ patients in group II had epileptiform disturbances in EEG. Therapy of these patients was changed to antiepileptic drugs what entailed significant improvement of speech, social skills, repetitive patterns of behavior.

Conclusion. Disturbances in the EEG are a fullfledged but not subsidiary symptom in the diagnosis and choice of tactics for treating ASD in children. There is a clear relationship between ASD and the epileptiform disturbances in the EEG.

\section{Result of EEG study}

n discharges in EEG without discharges in EEG

without normal period

normal period

\section{9}

North-Western State Medical University named after I.I.Mechnikov.

Kirochnaya st, 41

Russian Federation, Saint-Petersburg

Alexandr Sofronov, Ilya Zaytsev - psychiatry

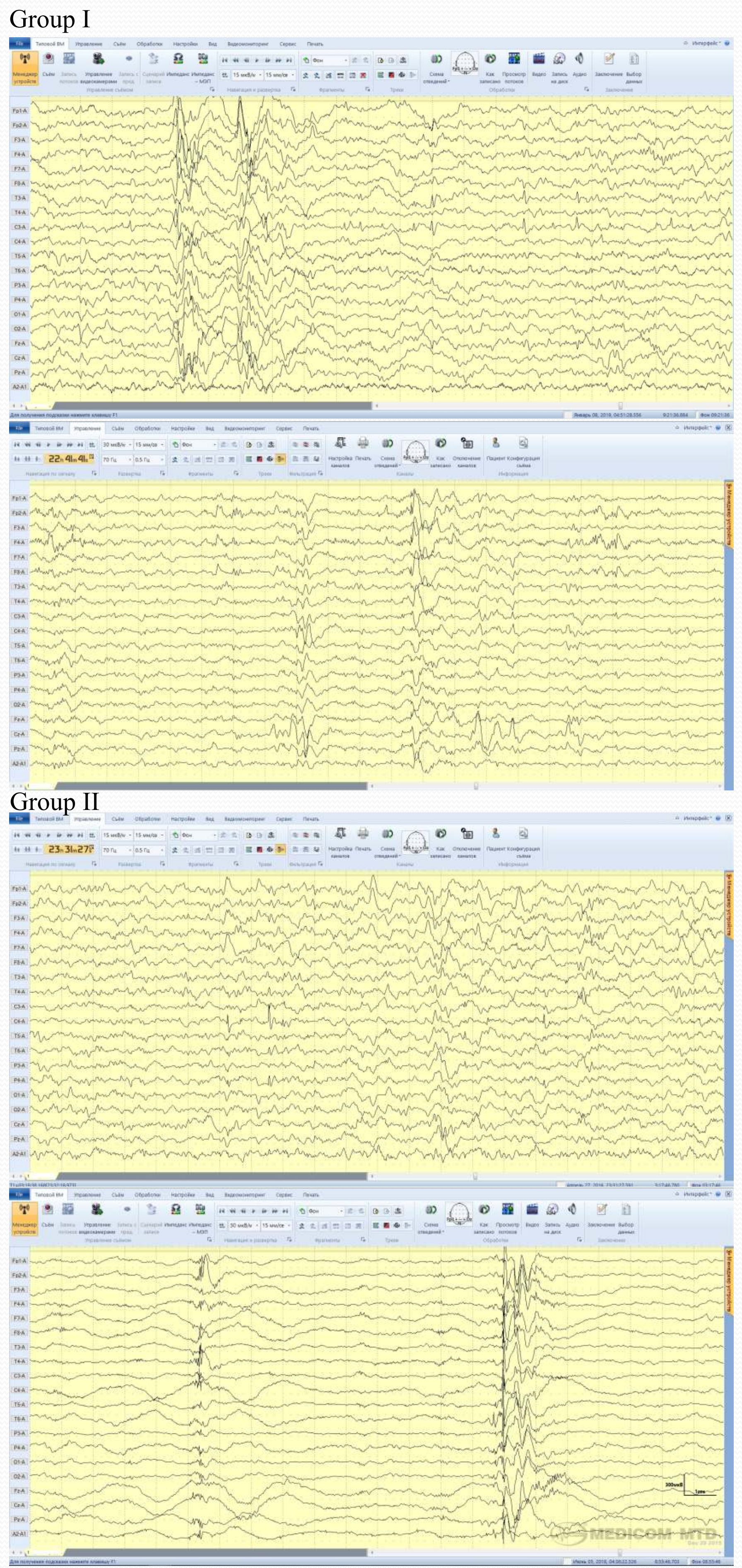

Copyright (C) 2019 Dmitry Zaytsev, Ilya Zaytsev, Nikolay Titov; Zaytsev's Psychoneurological cetnetr ltd.

Russian Federation, Saint-Petersburg, Ligovsky prospect, 50 liter F Tel./fax.: +7(812)764-27-10, e-mail: pnc-z@pncz.ru Alexandr Sofronov, Ilya Zaytsev;

North-Western State Medical University named after I.I.Mechnikov Russian Federation, Saint-Petersburg, Kirochnaya street, 41

Tel./fax.: +7(812)303-50-00, e-mail: dr.zaytsev.ilya@gmail.com 